\title{
OPTIMAL NONLINEAR CONTROL OF VEHICLE BRAKING TORQUES TO GENERATE PRACTICAL STABILIZING YAW MOMENTS
}

\author{
M. Jafari, M. Mirzaei* and H. Mirzaeinejad \\ Faculty of Mechanical Engineering, Sahand University of Technology \\ P.O.Box 51335-1996, Sahand new town, Tabriz, Iran \\ Email: mirzaei@sut.ac.ir
}

\begin{abstract}
In this paper, a new optimal strategy for differential braking is proposed to recover the vehicle stability in emergency maneuvers. Practical aspects of the problem are considered in the design of a control system with two layers. In the upper layer, an optimal nonlinear control law is analytically developed for calculating the external yaw moment and the distributed braking forces. The optimal property of the control law provides the possibility of reducing the control input to the lowest possible value to avoid undesirable effects. At the same time, considering the force capacity of each tire, a practical stabilizing yaw moment can be calculated by adjusting the weighting ratio as a free parameter of the control law. In the lower layer, a nonlinear wheel slip tracking controller is designed for the front wheels to generate the required braking forces of the upper layer. The simulation studies carried out by a nonlinear eight-degrees-of-freedom (8DOF) vehicle model indicate that the proposed control system can improve vehicular handling and stability with a practical external yaw moment and reduced differential braking forces.
\end{abstract}

Keywords: Differential braking; optimal nonlinear controller; tire force capacity; prediction.

\section{INTRODUCTION}

During severe turning maneuvers, the decrease of the internal yaw moment generated by tire lateral forces when the sideslip angle increases is the basic cause of a vehicle's unstable motion (spin motion). In these maneuvers, the effectiveness of the vehicle steering angle in generating the restoring yaw moment is significantly reduced because of tire force saturation which is considered as a hard nonlinearity [1, 2]. Direct yaw moment control (DYC) is an effective method introduced to control vehicle stability and steerability in nonlinear regimes [1-3]. A practical approach to generating a required external yaw moment, independent of lateral forces and steering angle, is the transverse distribution of the vehicle braking force between the left and right wheels. This strategy is known as differential braking (DB) and can be achieved by controlling the longitudinal slip of each individual wheel through the common anti-lock braking system [4]. DYC and DB are considered as the upper and lower layers of the vehicle dynamic control (VDC) or electronic stability program (ESP) system respectively [5]. Several control methods for calculation of stabilizing external yaw moments can be found in the literature. Some researchers have developed optimal control laws for DYC. In this context, the well-known linear quadratic regulator (LQR) theory has been used to control vehicle handling and stability [1, 6-8]. A predictive optimal yaw stability 
controller based on a linearized vehicle model that was discretized via a bilinear transformation was presented by Anwar [9]. Such methods apply linear vehicle models to find control laws, whereas the nonlinear characteristic of vehicle dynamics and tire forces is the most important issue in the controller design for DYC. Sliding control methods have been frequently applied in stability control because of their potential to cope with nonlinearities and intrinsic robustness [8, 10-13]. In other works, a fuzzy logic stability control [14] and a robust velocity-dependent control law [15] have been used for yaw moment control. In the control methods mentioned above, optimization is not used as the main procedure for determining control laws, whereas the external yaw moment is considered as an expensive control input and should be kept at a minimum value because of its undesirable side-effects. Excessive use of control effort causes a vehicle to slow down because a corrective yaw moment is applied to the vehicle through the brakes. This effect must be kept to a minimum so that the driver can feel supported rather than overruled. Furthermore, tire life and other components such as brake pad and disc are also shortened because of extra braking. One approach to limiting the excessive use of external yaw moments is integrating and coordinating DYC and steering control systems [16-18].

In this paper, in answer to the control requirements of the DYC system, an optimal nonlinear yaw moment control law in the closed form is developed; it is based on the response prediction of a nonlinear four-wheel vehicle model. The optimal nonlinear control law is given in an analytical closed form that is easy to solve and online numerical computations in optimization are not necessary, in contrast to the classic optimal control theories such as LQR. This is an extension of the previous control law designed by the author's research team based on the (2DOF) bicycle model [19]. However, in that work, the reduced external yaw moment was directly applied to the vehicle model without transformation to the differential braking forces. Therefore, it cannot be determined whether the calculated external yaw moment can be generated in practice or not. From the practical perspective, the external yaw moment calculated from the control law has to be generated by differential braking through the main parts of the common anti-lock braking system. The calculated yaw moment must remain below the maximum admissible value determined by the force capacity of each tire, which is different because of the effects of normal load transfer and nonlinear tire characteristics. Even if no control saturation occurs, the external yaw moment should be kept as low as possible because of the undesirable side-effects mentioned before. Accordingly, determining how much the stabilizing external yaw moment should be reduced to generate the practical differential braking forces is the main aim of the present study. To achieve this aim, a control system with two layers is designed. In the upper layer, an optimal yaw moment control law is developed. In this layer, the expensive control approach is considered in which the minimized performance index consists of a linear combination of tracking error and control energy. Adjusting the weighting factors as the free parameters of the control law provides the possibility of reducing the external yaw moment within the admissible range by considering the force capacity of each tire, at the cost of admissible tracking error. The calculated admissible yaw moment is converted to the differential braking force between the front left and right wheels. In the lower layer, depending on the longitudinal dynamics of the wheel, a slip controller is designed to generate the braking force of each tire by regulating their longitudinal slip. Finally, to evaluate the performance and robustness of the proposed control system and the improvement of vehicle handling and stability, simulation studies have been performed with the 8DOF nonlinear vehicle model. The results are 
compared with those obtained by the LQR controller as a classic linear optimal control method. The yaw rate tracking error and control energy are compared with regard to the practical aspects of the vehicle system.

\section{MODELING OF THE VEHICLE SYSTEM}

In this paper, an 8DOF vehicle model validated by experimental results [20] has been constructed as the vehicle plant model. Since this model is too complicated for use in the controller design, a simpler vehicle model referred to as the design model is employed for development of the control law. In this paper, in accordance with the control system requirements, a nonlinear 2DOF model that captures the essential features of vehicle lateral dynamics is used to design the yaw moment controller.

\section{Nonlinear 8DOF model for simulation}

The 8DOF vehicle model used for simulation studies is shown in Figure 1. All notations are described in Appendix 1.
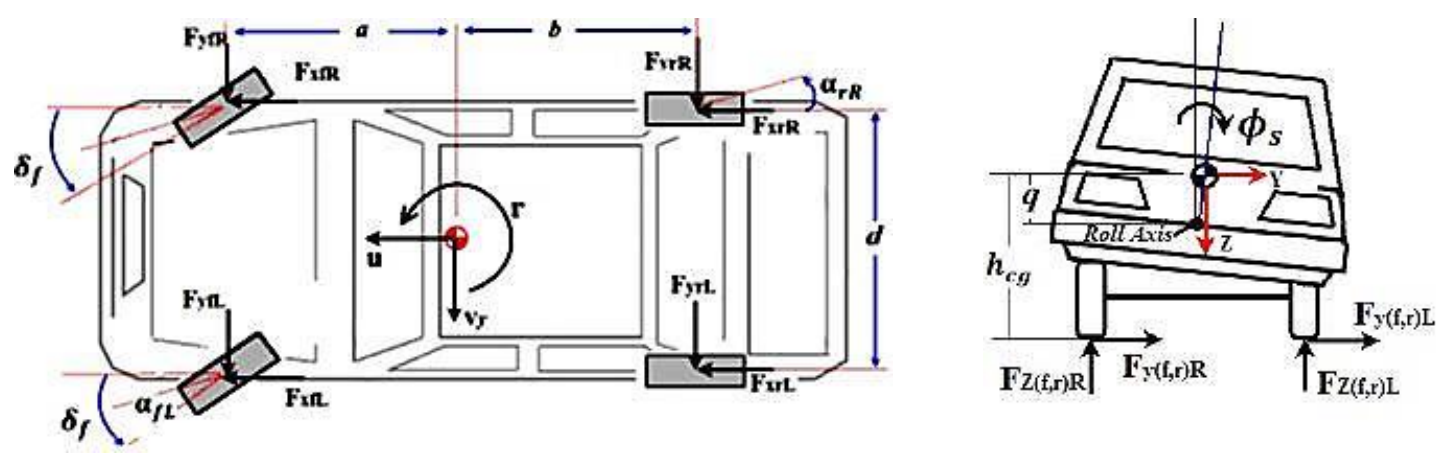

Figure 1. Vehicle free body diagram.

The governing equations of vehicle motion including the longitudinal, lateral, yaw and roll dynamics can be expressed as follows:

$$
\begin{gathered}
m \dot{u}=m v_{y} r+F_{x f R}+F_{x f L}+F_{x r R}+F_{x r L} \\
m \dot{v}_{y}+m_{s} q \dot{p}=-m u r+F_{y f R}+F_{y f L}+F_{y r R}+F_{y r L} \\
\mathrm{I}_{\mathrm{zz}} \dot{\mathrm{r}}-\mathrm{I}_{\mathrm{xz}_{\mathrm{s}}} \dot{\mathrm{p}}=\mathrm{a}\left(\mathrm{F}_{\mathrm{yfR}}+\mathrm{F}_{\mathrm{yfL}}\right)-\mathrm{b}\left(\mathrm{F}_{\mathrm{yrR}}+\mathrm{F}_{\mathrm{yrL}}\right)+\mathrm{d} / 2\left(\mathrm{~F}_{\mathrm{xfL}}+\mathrm{F}_{\mathrm{xrL}}\right)-\mathrm{d} / 2\left(\mathrm{~F}_{\mathrm{xfR}}+\mathrm{F}_{\mathrm{xfR}}\right) \\
I_{x x_{s}} \dot{p}+m_{s} q \dot{v}_{y}-I_{x z_{s}} \dot{r}=-m_{s} q u r+m_{s} g \sin \varphi_{s}-K_{\varphi} \varphi_{s}-C_{\varphi} p
\end{gathered}
$$

In the above equations, the forces in the $\mathrm{x}$ and $\mathrm{y}$ coordinates of the vehicle are related to the longitudinal and lateral forces of each wheel as follows:

$$
\begin{aligned}
& F_{x i}=F_{t i} \cos \delta_{w i}-F_{s i} \sin \delta_{w i} \\
& F_{y i}=F_{t i} \sin \delta_{w i}-F_{s i} \cos \delta_{w i} i=f R, f L, r R, r L
\end{aligned}
$$

where the steering angle of the front and rear wheels including roll steer coefficients are $\delta_{w f}=\delta_{f}+k_{r s f} \phi_{s}$ and $\delta_{w r}=k_{r s r} \phi_{s}$, respectively. 
The tire forces are modeled by the nonlinear Dugoff's tire model which is based on the friction ellipse idea [21]. In this model, the relations for longitudinal and lateral forces of each tire are as follows:

$$
F_{t i}=\frac{C_{i} \lambda_{w i}}{1-\lambda_{w i}} f(S), \quad F_{s i}=\frac{C_{\alpha} \tan \alpha_{i}}{1-\lambda_{w i}} f(S), \quad i=f R, f L, r R, r L
$$

where

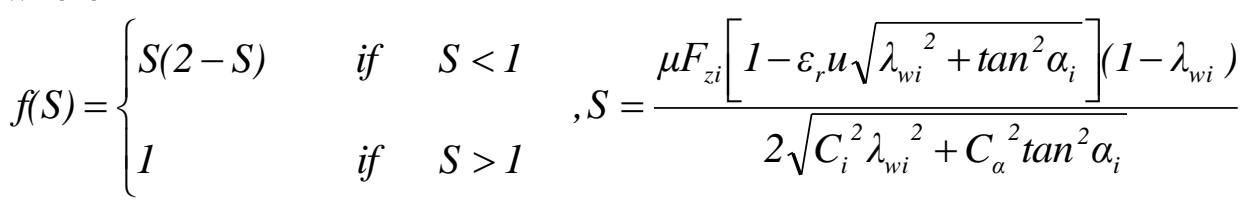

Also, the rotational dynamics of each wheel during braking is modelled as follows:

$$
\dot{\omega}_{i}=\frac{1}{I_{w i}}\left[R F_{b i}-T_{b i}\right]
$$

\section{Nonlinear 2DOF model for controller design}

The nonlinear 2-DOF model with constant speed has been used to design the yaw moment controller. The governing equations of motion for this model are as follows:

$$
\begin{aligned}
& \dot{v}_{y}=\frac{1}{m}\left[-m u r+F_{s f R} \cos \delta_{f}+F_{s f L} \cos \delta_{f}+F_{s r R}+F_{s r L}\right] \\
& \dot{r}=\frac{1}{I_{z z}}\left[a\left(F_{s f R}+F_{s f L}\right) \cos \delta_{f}-b\left(F_{s r R}+F_{s r L}\right)\right]+\frac{1}{I_{z z}} M_{z}
\end{aligned}
$$

\section{Desired model for yaw rate}

In order to compensate the loss of vehicle stability due to nonlinear characteristics of tire forces, a linear 2DOF vehicle plane model with appropriate limitations within physical constraints is presented as a desired model to be followed by the controller. In this model is expressed as follows [7]:

$$
\frac{r_{d}(s)}{\delta_{f}(s)}=G_{R} \frac{1}{1+T_{t} s}
$$

where,

$$
\begin{gathered}
G_{R}=\frac{b_{1} a_{21}-b_{2} a_{11}}{a_{11} a_{22}+a_{12} a_{21}}, \quad b_{1}=\frac{2 C_{\alpha f}}{m u}, \quad b_{2}=\frac{2 a C_{\alpha f}}{I_{z z}}, \quad a_{11}=-2 \frac{C_{\alpha f}-C_{\alpha r}}{m u} \\
a_{12}=2 \frac{b C_{\alpha r}-a C_{\alpha f}}{m u^{2}}-1, \quad a_{21}=2 \frac{b C_{\alpha r}-a C_{\alpha f}}{I_{z z}}, \quad a_{22}=-2 \frac{b^{2} C_{\alpha r}-a^{2} C_{\alpha f}}{I_{z z} u}
\end{gathered}
$$


The following limitation should be applied on the steady state value of Eq. (11):

$$
r_{s s}=\left\{\begin{array}{lr}
G_{R} \delta_{f} \quad \text { if } & \left|G_{R} \delta_{f}\right|<\frac{\mu g}{u} \\
\frac{\mu g}{u} \operatorname{sign}\left(G_{R} \delta_{f}\right) & \text { otherwise }
\end{array}\right.
$$

In the above relations, $r_{d}$ is the desired yaw rate and $T_{t}$ is the time constant of yaw rate.

\section{CONTROL SYSTEM DESIGN}

The structure of the proposed control system with two layers can be seen in the Figure 2. In the upper layer, a yaw moment control law is developed in an optimal approach. Then, the calculated admissible yaw moment is converted to the differential braking force between the front left and right wheels. In the lower layer, a nonlinear wheel slip controller is designed to generate the braking force calculated from the upper layer.

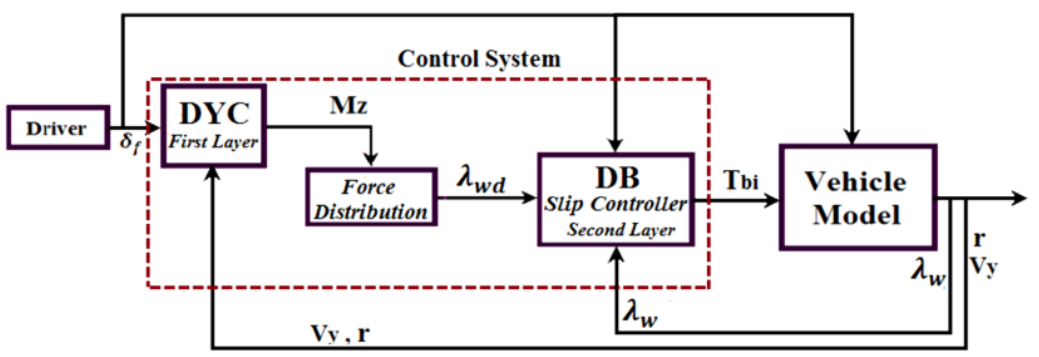

Figure 2. Structure of vehicle dynamic.

\section{Development of yaw moment control law (First layer)}

The nonlinear 2DOF vehicle model described by Eqs. and can be written in the state space form if the yaw rate is considered as the output of the system:

$$
\begin{aligned}
& \dot{x}_{1}=f_{1}\left(x, \delta_{f}\right) \\
& \dot{x}_{2}=f_{2}\left(x, \delta_{f}\right)+\frac{1}{I_{z z}} M_{z} \\
& y=x_{2}
\end{aligned}
$$

where $x=\left[\begin{array}{ll}v_{y} & r\end{array}\right]^{T}$ is the state vector and $y$ is the output. The external yaw moment $M_{z}$ and the front wheel steering angle $\delta_{f}$ represent the control input and disturbance input, respectively. The nonlinear Dugoff's tire model has been incorporated in $f_{1}$ and $f_{2}$. In order to develop an optimal nonlinear yaw moment control law based on the prediction, a pointwise minimization performance index that penalizes both the tracking error of 
yaw rate at the next instant, $r(t+h)$, and the current control expenditure, $M_{z}(t)$, is considered as follows:

$$
J_{1}\left[M_{z}(t)\right]=\frac{1}{2} w_{1}\left[r(t+h)-r_{d}(t+h)\right]^{2}+\frac{1}{2} w_{2}\left[M_{z}^{2}(t)\right]
$$

The predictive period $h$ is a real positive number. Now, in order to expand the performance index (Eq. (13)) as a function of control input, it is necessary to predict the yaw rate response for the next time interval by Taylor series. First approximate $r(t+h)$ by a $k$ th-order Taylor series at $t$ according to:

$$
r(t+h)=r(t)+h \dot{r}(t)+\frac{h^{2}}{2 !} \ddot{r}(t)+\ldots+\frac{h^{k}}{k !} r^{(k)}(t)
$$

The expansion order $k$ is determined by the relative degree of the nonlinear system $[22,23]$. According to the state equations of the system, since the vehicle yaw rate dynamics has a well-defined relative degree, $\rho=1$, the first-order Taylor series is sufficient for the expansion of yaw rate and its desired value as follows:

$$
\begin{gathered}
r(t+h)=r(t)+h\left(f_{2}+\frac{1}{I_{z z}} M_{z}\right) \\
r_{d}(t+h)=r_{d}(t)+h \dot{r}_{d}(t)
\end{gathered}
$$

Now, by substituting Eqs. (15) and (16) into (13), the expanded performance index can be obtained as a function of control input. The necessary condition for optimality is:

$$
\frac{\partial J_{1}}{\partial M_{z}}=0
$$

which leads to:

$$
M_{z}(t)=\frac{I_{z}}{h} \frac{1}{1+\lambda I_{z}^{2} h^{-2}}\left[e_{1}+h\left(f_{2}-\dot{r}_{d}\right)\right]
$$

where $e_{1}$, is the current tracking error.

$$
e_{1}=r-r_{d}
$$

and $\lambda$ is the weighting ratio

$$
\lambda=\frac{w_{2}}{w_{1}} \geq 0
$$

If we increase the weighting ratio $\lambda$, the external yaw moment can be reduced at the cost of some tracking errors in the yaw rate. When $\lambda=\infty$, then $M_{z}=0$. This case is 
considered as the expensive control and corresponds to the uncontrolled system. In contrast, the cheap control is related to the case of $\lambda=0$ in which there is no reduction in control input. In practice, the calculated external yaw moment is implemented through braking one of the left and one of the right wheels. Because of the high force capacity and dominant effect of the front tires during cornering and braking, the required braking force is applied to one of the front wheels [15]. Therefore, the necessary braking force can be calculated as follows:

$$
F_{b}=2 / d M_{z}
$$

In relation to the algorithm shown in Figure 3, the braked wheel is selected according to the yaw rate and yaw rate tracking error.

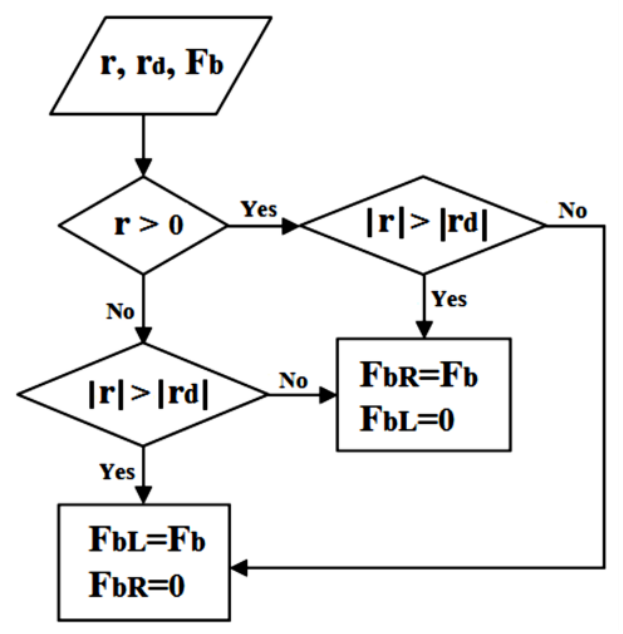

Figure 3. Distribution of the braking forces.

Now, substituting Eq. (18) into Eq. (21) yields:

$$
F_{b}(t)=\frac{2}{d} \frac{I_{z}}{h} \frac{1}{1+\lambda I_{z}^{2} h^{-2}}\left[e_{1}+h\left(f_{2}-\dot{r}_{d}\right)\right]
$$

To find a suitable criterion for adjusting the weighting ratio $\lambda$, the force capacity of the braked wheel should be considered. The following constraint indicating the tire workload should always be satisfied for the braked wheel.

$$
F_{b}^{2}+F_{s i}^{2} \leq\left(\mu F_{s i}\right)^{2} \quad i=f R \text { or } f L
$$

The minimum value of the weighting ratio $\lambda$ is selected by satisfying the equality constraint of Eq. (23). If the weighting ratio is increased, the control input is decreased even more. It should be noted that the value of the weighting ratio can be increased to some extent; otherwise the yaw rate cannot follow the behavior of the reference model adequately and the vehicle remains unstable. In the next section, a wheel slip controller is designed for the braking torque to generate the braking force calculated from Eq. (22). 


\section{Development of the wheel slip control law (Second layer)}

In order to generate the required external braking force, the corresponding wheel slip of the braked wheel is first calculated by the inverse of a simplified Dugoff 's tire model. This wheel slip, called desired wheel slip, will be tracked by the slip controller. Differentiating longitudinal wheel slip during braking, $\lambda_{w i}=1-\frac{R \omega_{i}}{u}$, with respect to the time and using the equations of motions of the wheel model yields [24]:

$$
\dot{\lambda}_{w}=g(x)+\left(\frac{R T_{b}}{u I_{w}}\right)
$$

The nonlinear Dugoff's tire model has been incorporated in $g(x)$. In the same manner as before, the first-order Taylor series will be sufficient for the expansion of wheel slip and its desired value for the next time interval:

$$
\begin{gathered}
\lambda_{w}(t+h)=\lambda_{w}(t)+h\left(g(x)+\frac{R}{u I_{w}} T_{b}\right) \\
\lambda_{w d}(t+h)=\lambda_{w d}(t)+h \dot{\lambda}_{w d}(t)
\end{gathered}
$$

Now, a performance index that penalizes the next instant tracking error is considered in the following form:

$$
J_{2}\left[T_{b}(t)\right]=\frac{1}{2}\left[\lambda_{w}(t+h)-\lambda_{w d}(t+h)\right]^{2}
$$

After substitution of Eqs. (25) and (26) into Eq. (27), the necessary condition for optimality can be applied:

$$
\frac{\partial J_{2}}{\partial T_{b}}=0
$$

which leads to:

$$
T_{b}=\frac{u I_{w}}{R h}\left[e_{2}+h\left(g-\dot{r}_{d}\right)\right]
$$

Where

$$
e_{2}=\lambda_{w}-\lambda_{w d}
$$

\section{RESULTS AND DISCUSSION}

Computer simulations have been carried out to show the performance of the proposed control system. How to adjust the external yaw moment in a practical range according to the force capacity of tires is also investigated for a case study vehicle by using an 8- 
DOF nonlinear vehicle model. The model parameters employed in this study are given in [18]. The responses of the uncontrolled vehicle are compared with those obtained by the cheap control version of the proposed control system in which the weighting ratio is taken to be zero in calculating the external yaw moment. A severe lane-change maneuver is considered in this study. In this maneuver, the vehicle travels along a straight path on a level low-friction road $(\mu=0.4)$ at a constant speed of $70 \mathrm{~km} / \mathrm{h}$ and the steering angle input, as shown in Figure 4(a), performs a sequence of positive and negative step inputs. To evaluate the robustness of the designed control system, $20 \%$ uncertainty in the total mass of the vehicle and $10 \%$ uncertainty in the friction coefficient are assumed.

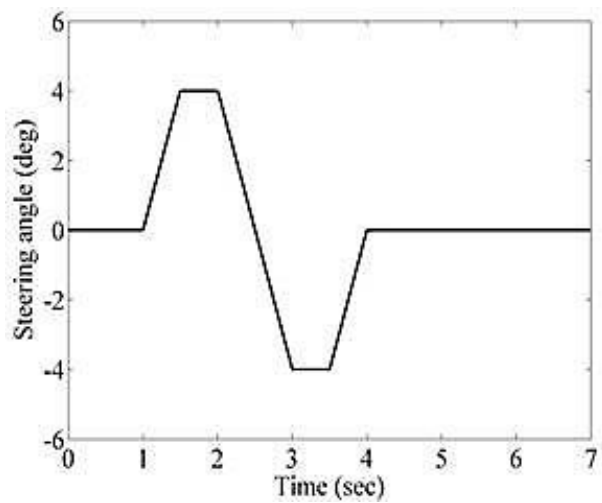

(a)

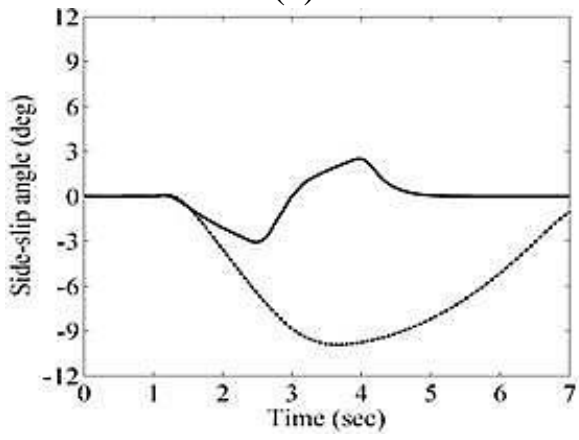

(c)

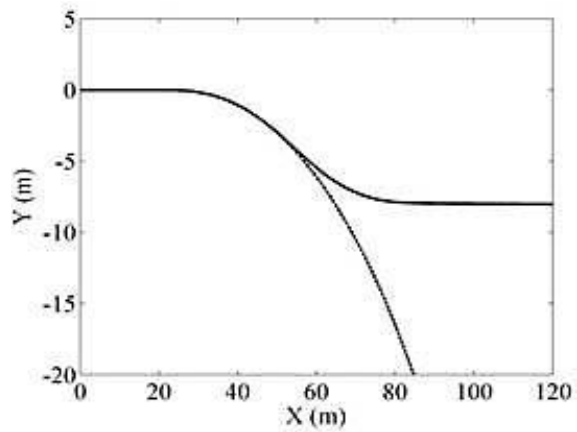

(e)

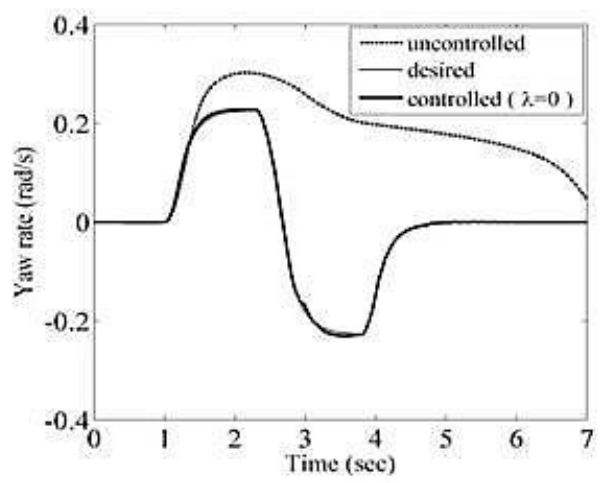

(b)

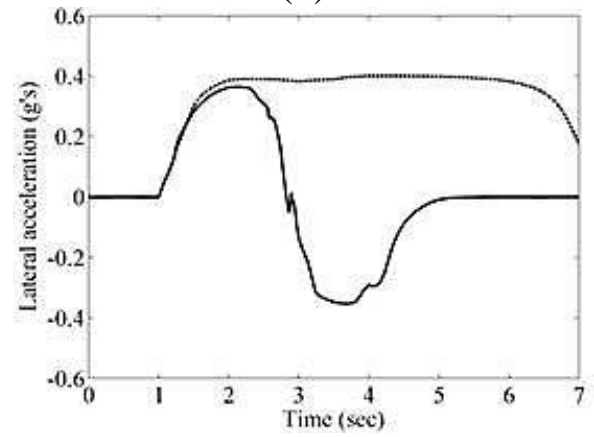

(d)

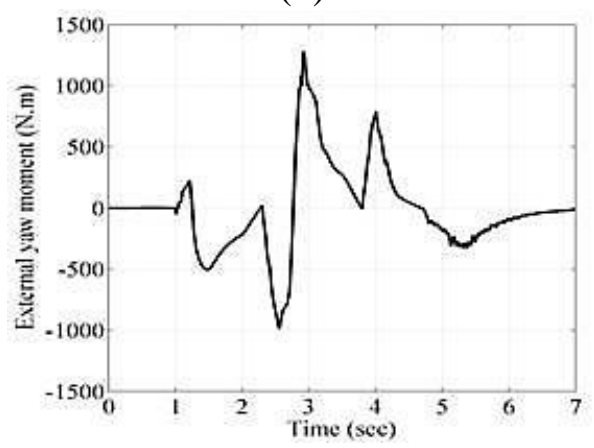

(f)

Figure 4. Simulation results of a lane-change maneuver with and without control $(\lambda=0)$.

The results in Figure 4 indicate that the uncontrolled vehicle cannot complete the lane change path because of the saturation of tire lateral forces when the slip angle 
increases as mentioned in ref. [1-3]. In contrast, the control system can successfully stabilize the vehicle by applying the external yaw moment depicted in Figure 4(f). It should be noted that there is no reduction in the external yaw moment by applying the zero weighting ratio $(\lambda=0)$. In this case, as seen in Figure 4(b), a relatively perfect tracking of yaw rate is achieved through a small value of prediction time $(h=0.03 \mathrm{sec})$ in the presence of modeling uncertainty. However, it is not certain whether the external yaw moment can be generated in practice or not. The normalized work load of the front tires is shown in Figure 5. As can be seen, the workloads of tires exceed one. This means that the required braking force of each tire is more than its force capacity and cannot be generated in practice. In fact, the calculated external yaw moment is beyond the maximum admissible value and has to be limited. To overcome the mentioned problem, the expensive control version of the proposed control system is employed. For illustration of this important feature of the control system, the weighting ratio is adjusted to $\lambda=1.2 \times 10^{-9}$. The time responses of the vehicle system together with the calculated external yaw moment are depicted in Figure 6. The normalized workload of tires is shown in Figure 7.
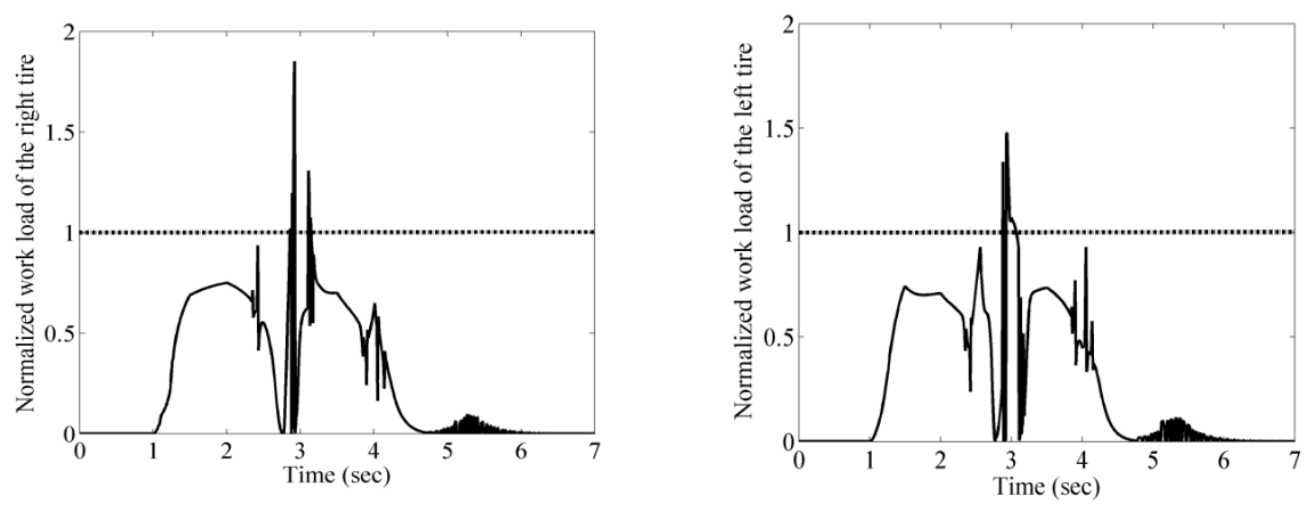

Figure 5. Normalized workload of the front tires.

As shown in Figures 6 and 7, the calculated external yaw moment and the braking forces are significantly decreased by applying the weighting ratio such that the workloads of tires are below one. This means that the required braking force of each tire is in a practical range and can be generated. As a result of reducing the control input by the specified $\lambda$, the yaw rate tracking error is increased as shown in Figure 6(a). However, this error is acceptable so that the vehicle, as shown in Figure 6(d), can perform the maneuver successfully. Also, the braking torque of the front tires is shown in Figure 8. The results show the good performance of the wheel slip controller in the lower layer. At the end of the simulation study, the dynamic performance of the proposed controller was compared with that of a classic optimal controller like LQR. The LQR method is based on a linear vehicle model and has been widely used in the literature for the design of DYC. For better comparison, the weighting factors of both controllers are regulated in such a way that the same control effort can be approximately obtained. As seen in Figure 9(a), the yaw rate responses of the vehicle with both controllers follow the behavior of the desired value. 


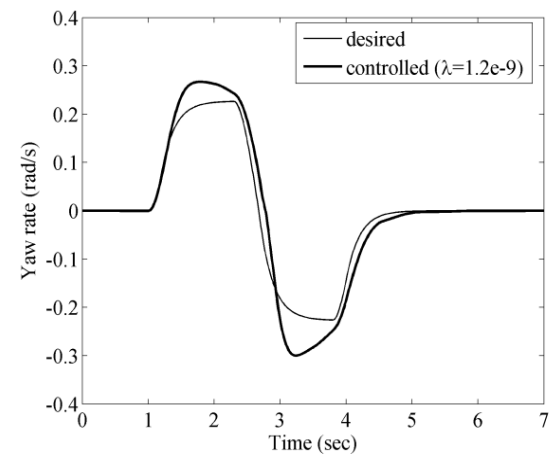

(a)

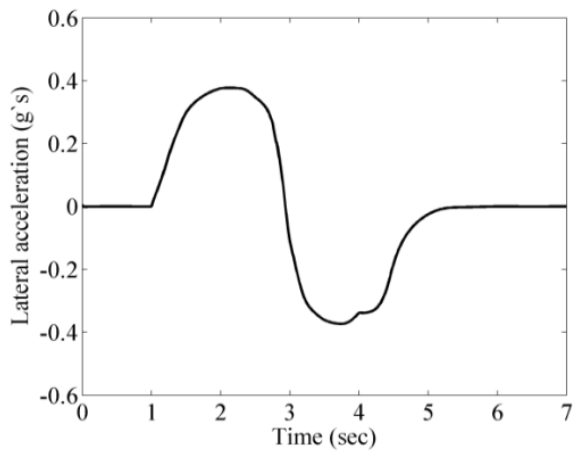

(c)

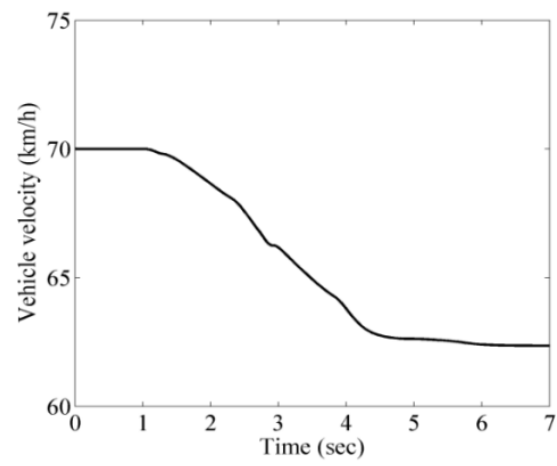

(e)

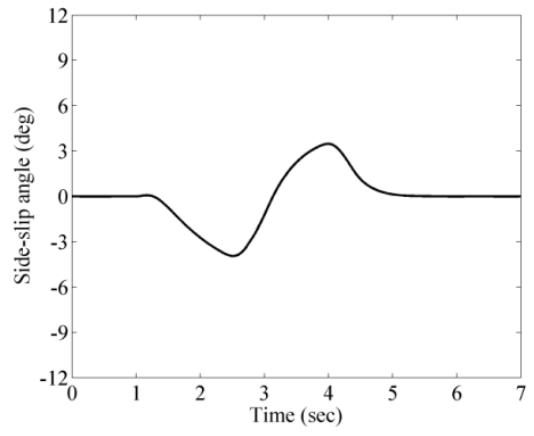

(b)

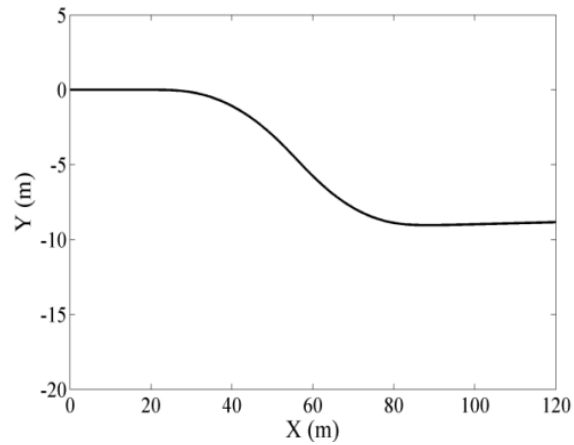

(d)

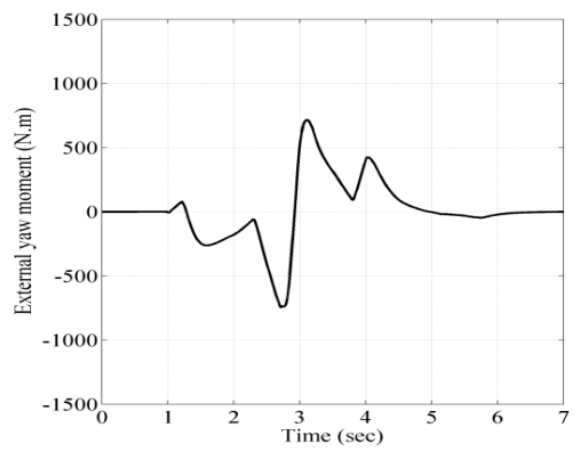

(f)

Figure 6. Simulation results of a lane-change maneuver with expensive control.
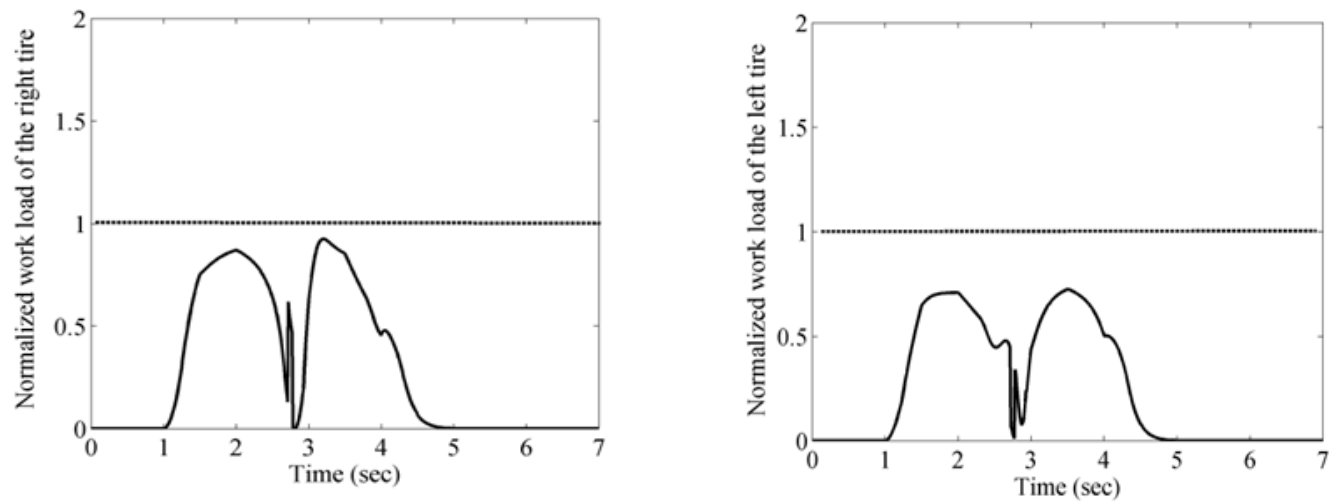

Figure 7. Normalized workload of the front tires. 


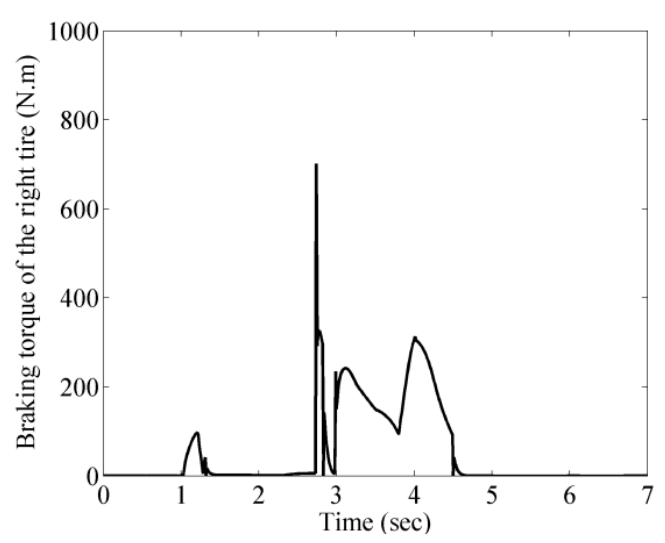

(a)

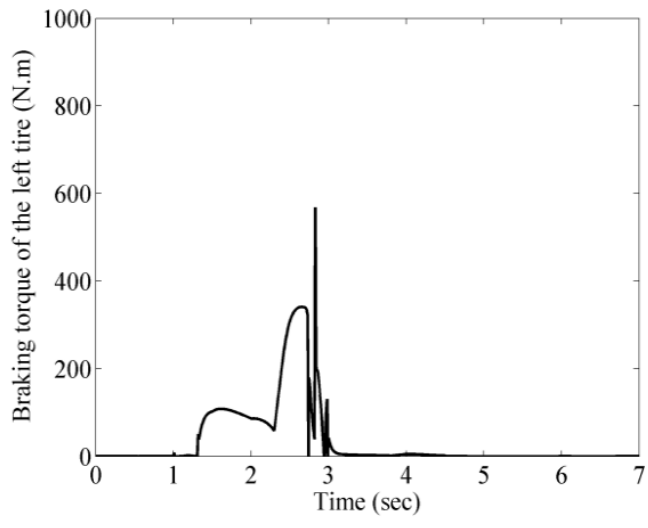

(b)

Figure 8 . Braking torque of the front tires designed in the lower layer.

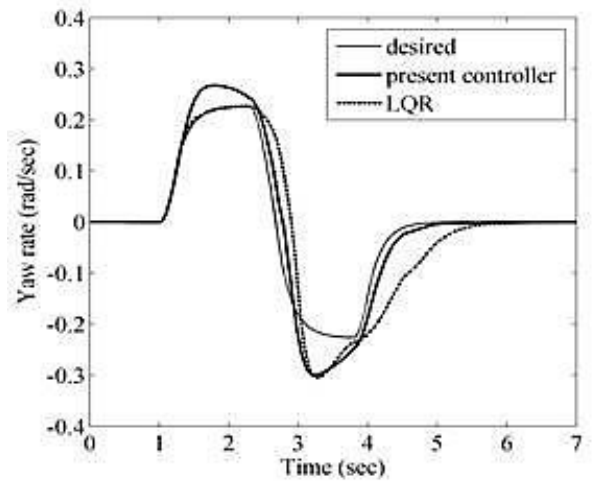

(a)

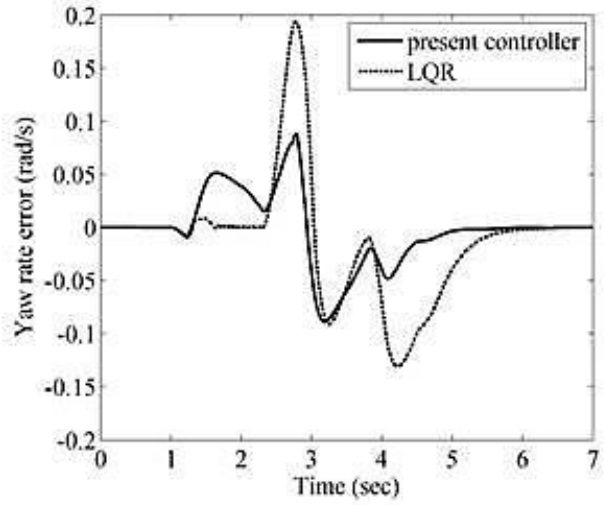

(b)

Figure 9. Comparison of the yaw rate tracking performance of the present controller and the LQR.

The yaw rate errors are also shown in Figure 9(b). The comparison of these figures indicates that the proposed optimal nonlinear controller follows the desired model through smaller tracking errors and lower control energy rather than the LQR controller during the control process. These results are confirmed by the performance indexes given in Table 1. The results show that the small tracking error is obtained with the LQR during the times of 1.5 to $2 \mathrm{sec}$ and produces the oscillation on the control input in this time interval. However, Figure 10 indicates that the normalized workload of the right tire exceeds one during the mentioned time interval by the LQR controller. This is because the LQR controller, which is based on the linear vehicle model, is engaged with a nonlinear vehicle model. Therefore, in the linear regimes and at the beginning of the maneuver, it performs better than the proposed nonlinear method. However, it deteriorates in the second part of the lane change maneuver which is a severe condition. However, the proposed nonlinear controller can better predict the vehicle response and gives a more satisfactory performance every time. Besides the above simulation results, the easier calculation and implementation of the proposed optimal nonlinear control law are the other advantages it has over the LQR controller. Also, the adjustment of the free parameters of the proposed controller and its management are effortless for designers, unlike the classic optimal control methods like LQR. 
Table 1. Comparison of dynamic performances of two controllers.

\begin{tabular}{ccc}
\hline Method & Proposed controller & LQR controller \\
\hline $\int_{0}^{7} M_{z}^{2} d t \times 10^{-5}$ & 6.58 & 6.59 \\
$\int_{0}^{7}\left(r-r_{d}\right)^{2} d t \times 10^{4}$ & 78 & 241 \\
\hline
\end{tabular}

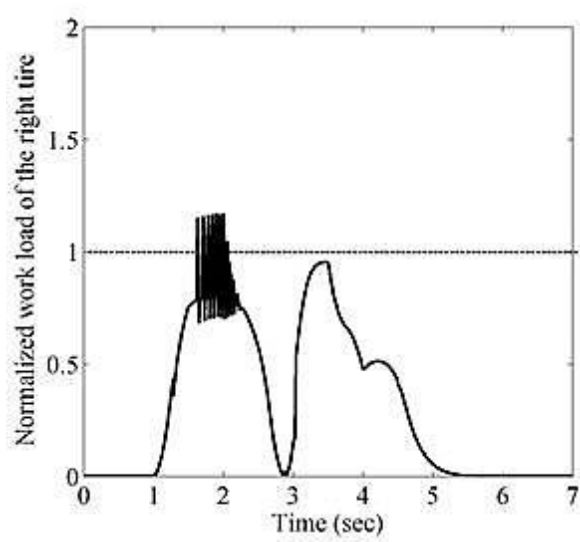

(a)

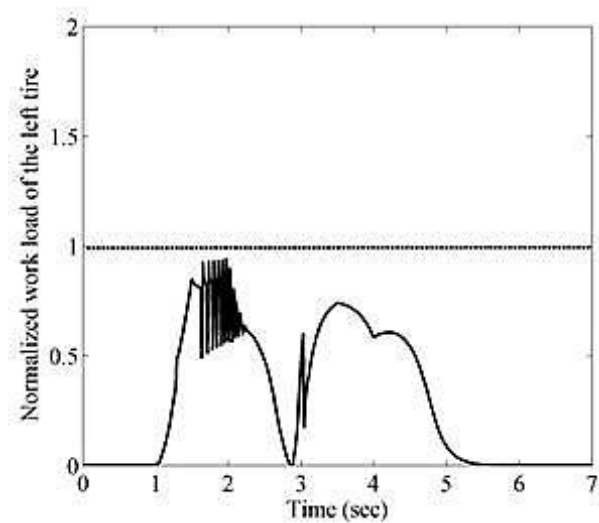

(b)

Figure 10. Normalized workload of the front tires with the LQR controller.

\section{CONCLUSIONS}

Using the lowest differential braking forces in the practical range to recover vehicle stability in nonlinear regimes can be considered as an important advantage. In this respect, a new optimal strategy for differential braking is proposed which considers the force capacity of tires. The obtained results indicate that the proposed control system can improve vehicle handling and stability by means of a reduced practical external yaw moment and differential braking forces. Finally, comparison of the results shows the efficiency of the proposed controller is better than that of the LQR controller.

\section{ACKNOWLEDGMENTS}

The authors would like to thanks to Sahand University of Technology, Iran for financial support and laboratory facilities.

\section{REFERENCES}

[1] Zheng S, Tang H, Han Z, Zhang Y. Controller design for vehicle stability enhancement. Control Engineering Practice. 2006;14:1413-21.

[2] $\mathrm{Li} \mathrm{D}, \mathrm{Du} \mathrm{S}, \mathrm{Yu} \mathrm{F}$. Integrated vehicle chassis control based on direct yaw moment, active steering and active stabiliser. Vehicle System Dynamics. 2008;46:341-51.

[3] Shibahata Y, Shimada K, Tomari T. Improvement of vehicle maneuverability by direct yaw moment control. Vehicle System Dynamics. 1993;22:465-81.

[4] Goodarzi A, Esmailzadeh E. Design of a VDC system for all-wheel independent drive vehicles. Mechatronics, IEEE/ASME Transactions on. 2007;12:632-9. 
[5] Van Zanten AT, Erhardt R, Landesfeind K, Pfaff G. VDC systems development and perspective. SAE Technical Paper No. 980235; 1998.

[6] Esmailzadeh E, Goodarzi A, Vossoughi G. Optimal yaw moment control law for improved vehicle handling. Mechatronics. 2003;13:659-75.

[7] Mirzaei M. A new strategy for minimum usage of external yaw moment in vehicle dynamic control system. Transportation Research Part C: Emerging Technologies. 2010;18:213-24.

[8] Zulkarnain N, Zamzuri H, Mazlan SA. Ride and handling analysis for an active anti-roll bar: case study on composite nonlinear control strategy. International Journal of Automotive and Mechanical Engineering. 2014;10:2122-242.

[9] Anwar S. Generalized predictive control of yaw dynamics of a hybrid brake-bywire equipped vehicle. Mechatronics. 2005;15:1089-108.

[10] Yi K, Chung T, Kim J, Yi S. An investigation into differential braking strategies for vehicle stability control. Proceedings of the Institution of Mechanical Engineers, Part D: Journal of Automobile Engineering. 2003;217:1081-93.

[11] Heinzl P, Lugner P, Plochl M. Stability control of a passenger car by combined additional steering and unilateral braking. Vehicle System Dynamics. 2003;37:221-33.

[12] Naraghi M, Roshanbin A, Tavasoli A. Vehicle stability enhancement-an adaptive optimal approach to the distribution of tyre forces. Proceedings of the Institution of Mechanical Engineers, Part D: Journal of Automobile Engineering. 2010;224:443-53.

[13] Rahmat MS, Ahmad F, Mat Yamin AK, Aparow VR, Tamaldin N. Modeling and torque tracking control of permanent magnet synchronous motor (PMSM) for hybrid electric vehicle. International Journal of Automotive and Mechanical Engineering. 2013;7:955-67.

[14] Tahami F, Farhangi S, Kazemi R. A fuzzy logic direct yaw-moment control system for all-wheel-drive electric vehicles. Vehicle System Dynamics. 2004;41:203-21.

[15] Bang M, Lee S, Han C, Maciuca D, Hedrick J. Performance enhancement of a sliding mode wheel slip controller by the yaw moment control. Proceedings of the Institution of Mechanical Engineers, Part D: Journal of Automobile Engineering. 2001;215:455-68.

[16] Tavasoli A, Naraghi M, Shakeri H. Optimized coordination of brakes and active steering for a 4WS passenger car. ISA transactions. 2012;51:573-83.

[17] Yang X, Wang Z, Peng W. Coordinated control of AFS and DYC for vehicle handling and stability based on optimal guaranteed cost theory. Vehicle System Dynamics. 2009;47:57-79.

[18] Di Cairano S, Tseng HE, Bernardini D, Bemporad A. Vehicle yaw stability control by coordinated active front steering and differential braking in the tire sideslip angles domain. IEEE Transactions on Control Systems Technology. 2013;21:1236-48.

[19] Mirzaei M, Alizadeh G, Eslamian M, Azadi S. An optimal approach to nonlinear control of vehicle yaw dynamics. Proceedings of the Institution of Mechanical Engineers, Part I: Journal of Systems and Control Engineering. 2008;222:217-29.

[20] Smith DE, Starkey JM. Effects of model complexity on the performance of automated vehicle steering controllers: model development, validation and comparison. Vehicle System Dynamics. 1995;24:163-81. 
[21] Dugoff H, Fancher P, Segel L. An analysis of tire traction properties and their influence on vehicle dynamic performance. SAE Technical paper. 1970;700377.

[22] Lu P. Tracking control of nonlinear systems with bounded controls and control rates. Automatica. 1997;33:1199-202.

[23] Chen WH, Ballance DJ, Gawthrop PJ. Optimal control of nonlinear systems: a predictive control approach. Automatica. 2003;39:633-41.

[24] Mirzaeinejad H, Mirzaei M. A new approach for modelling and control of twowheel anti-lock brake systems. Proceedings of the Institution of Mechanical Engineers, Part K: Journal of Multi-body Dynamics. 2011;225:179.

\section{Appendix 1: Description of vehicle model parameters}

$m, m_{s}$

$l, h_{c g}$

$a, b$

$r, p, \phi$

$u, v_{y}$

$a_{y}$

$\beta$

$q, d$

$R, \omega_{i}, I_{w i}$

$I_{z z}$

$I_{x x_{s}}, I_{x z_{s}}$

$C_{i}, C_{\alpha}$

$K_{\phi}, C_{\phi}$

$\mu$

$\varepsilon_{r}$

$\delta_{f}$

$k_{r s f}, k_{r s r}$

$F_{x i}, F_{y i}$

$F_{t i}, F_{s i}$

$F_{b}$

$F_{z i}$

$\alpha_{i}, \lambda_{w i}$

$g$

$T_{i}, T_{b i}, F_{b i}$

$h$

$e_{1}, e_{2}$

$\lambda$

Subscripts

$f, \mathrm{r}$ front, rear total mass, sprung mass of the vehicle

the length of the wheel base, height of $\mathrm{CG}$ above ground

distance between center of gravity (CG) and the front, rear axle

yaw rate, roll rate and roll angle of the vehicle body

longitudinal velocity, lateral velocity

lateral acceleration

side-slip angle

distance from sprung mass CG to roll axis and wheel track width

wheel rolling radius, moment of inertia and angular speed of the

$i$ th wheel, $i=f R, f L, r R, r L i=f R, f L, r R, r L$

vehicle moment of inertia ( $\mathrm{Z}$ axis)

vehicle moment of inertia (roll axis), sprung mass product of inertia

longitudinal tire stiffness, cornering stiffness coefficient

roll axis stiffness and damping coefficients

the road coefficient of friction

the road adhesion reduction factor

steering input to the front wheels

roll steer coefficients

longitudinal and lateral forces of the ith wheel, $i=f R, f L, r R, r L$

tractive force, side force of the ith wheel, $i=f R, f L, r R, r L$

braking force

normal force of the ith wheel, $i=f R, f L, r R, r L$

the $i t h$ wheel slip angle, longitudinal wheel slip, $i=f R, f L, r R, r L$

gravity acceleration

drive torque, braking torque, braking force of the ith

wheel, $i=f R, f L, r R, r L$

predictive period

yaw rate tracking and longitudinal slip tracking errors

weighting ratio
$\mathrm{R}, \mathrm{L}$ right, left
d desired 